\title{
Ball Bearing Fault Diagnosis Using Supervised and Unsupervised Machine Learning Methods
}

\author{
V. Vakharia, V. K. Gupta and P. K. Kankar \\ Mechanical Engineering Discipline, PDPM Indian Institute of Information Technology, Design and Manufacturing \\ Jabalpur, Jabalpur-482005, India
}

(Received 29 September 2013; accepted 9 June 2014)

This paper deals with the approach of using multiscale permutation entropy as a tool for feature selection for fault diagnosis in ball bearings. The coefficients obtained from the wavelet transformation of the vibration signals of the bearings are used for the calculation of statistical parameters. Based on the minimum multiscale permutation entropy criteria, the best scale is selected and statistical parameters such as crest factor, form factor, and permutation entropy are calculated. Finally, the faults are classified by considering the statistical parameters and permutation entropy as features in supervised and unsupervised machine learning methods, such as a support vector machine and self-organizing maps, respectively. Results revealed that the multiscale permutation entropy-based feature extraction techniques provide higher classification accuracy in comparison to the other methodologies that have been proposed in previous published works. The methodology proposed in this paper also gives good results for unsupervised learning methods, i.e. self-organizing maps.

\section{NOMENCLATURE}

$C \quad$ penalty constant

$m$ embedding dimension

$M \quad$ total number of samples

$N \quad$ length of data

$s$ scale

$t_{1}$ time

$z \quad$ time series

$Y_{j}^{s} \quad$ coarse-grain time series

$w$ weight

$\xi_{i} \quad$ slack variable

$\pi \quad$ permutation pattern

$\tau$ time delay

\section{INTRODUCTION}

Techniques designed to monitor the conditions of rolling element bearings receive considerable attention from researchers across the globe. Faults in the bearings are the major source of the breakdown of machinery. When a defect in the surface of one bearing strikes the surface of another, impulsive force is generated. This effect has been exploited by several vibration analysis methods, as well as various signal processing techniques. ${ }^{1}$ Incipient fault diagnosis in rolling element bearings is essential for production efficiency and plant safety. Fault diagnosis depends mainly on the feature extraction techniques, because the signals carry dynamic information about the state of the machinery. The patterns of vibration signals, due to defects in various rotating parts, exhibit specific features. That is to say, faults can be identified by looking at pattern abnormalities in plant machinery and rotating parts. Signal processing techniques such as time domain, frequency domain, and combined time frequency domain (such as wavelet transformation) have been investigated by various researchers. ${ }^{2-5}$ Due to variations in friction, loading conditions, interaction of various rotating elements, and clearance and nonlinear stiffness of the bearings, 6,7 the vibration signals generated by machinery are often characterized by nonlinearity. Thus, nonlinear parameter estimation techniques have been widely used by many researchers. ${ }^{8-12}$ Numerous methods such as the correlation dimension ${ }^{13}$ and the Lyapunov exponent ${ }^{14}$ have been developed recently to detect nonlinearity. Entropy estimation is an important parameter for measuring system complexity. Analysis of the vibration signals generated from rotating machinery, using complexity measure such as approximate entropy ${ }^{15}$ and multiscale entropy, ${ }^{16}$ was used for the bearing fault diagnosis. Permutation entropy was introduced by Bandt and Pompe, ${ }^{18}$ and is a new nonlinear parameter estimation tool that was efficiently used for the fault diagnosis. ${ }^{17}$ By comparing neighbouring values, the complexity of a time series can be extracted using permutation entropy. Shannon entropy is useful for the estimation of the complexity of a time series based on a single scale, while multiscale permutation entropy is useful for calculating the complexity of a time series after comparing neighbouring values and entropy over multiple scales. Bandt and Pompe ${ }^{18}$ presented permutation entropy, as a parameter of average entropy, to describe the complexity of a time series. It should also be noted that feature vectors consisting of multiscale permutation entropy provide better information about physical phenomena such as the occurrence of faults in the rotor bearing system. ${ }^{19}$ The use of permutation entropy for chatter detection in the turning process, ${ }^{20}$ known as electroencephalography (EEG) signal analysis, ${ }^{21}$ has been used for chaotic time series. Signals obtained from complex mechanical systems that have several components are usually complicated. Approximate entropy and permutation entropy are based on a single scale, and are therefore inefficient in diagnosing the signals correctly. Multiscale permutation entropy was proposed by Costa, et al., ${ }^{22}$ and this concept was utilized by $\mathrm{Wu}$, et al. ${ }^{23}$ and Vakharia, et al., ${ }^{24}$ for bearing fault diagnosis and classification.

In this paper, raw vibration signals are used, and the concept of permutation entropy is utilized for the selection of scale. Wavelet coefficients are calculated by considering coiflet as 